J Nutr Health Aging. 2013 September ; 17(9): 738-739. doi:10.1007/s12603-013-0397-9.

\title{
COGNITIVE FRAILTY
}

\author{
A.S. BUCHMAN ${ }^{1,2}$ and D.A. BENNETT ${ }^{1,2}$ \\ ${ }^{1}$ Rush Alzheimer's Disease Center, Rush University Medical Center, Chicago, IL \\ ${ }^{2}$ Neurological Sciences, Rush University Medical Center, Chicago, IL
}

While the clinical hallmark of Alzheimer's disease (AD) is progressive loss of memory and other cognitive abilities, there is increasing recognition that persons with $\mathrm{AD}$ also exhibit changes in muscle bulk and body composition, strength and mobility, suggesting that many older persons with AD may be frail (1-7). Recent data suggest that changes in the motor system including reduced strength and walking speed and changes in body composition can antedate the onset of dementia $(1,3-6,8)$. Thus, while most studies examine late-life cognitive impairment and physical frailty separately, these findings raise the possibility that physical frailty, whose core features include loss of strength and muscle bulk, slowed gait and fatigue, share a common etiopathogensis with late-life cognitive impairment. In fact, recent studies suggest that both progressive physical frailty and cognitive decline in older adults are associated with common neuropathologies including post-mortem AD, Parkinson's disease (PD) and cerebrovascular disease (CVD) pathologies even in individuals without clinically diagnosed dementia, PD or stroke $(9,10)$. Further, we recently reported that neuronal density in the locus coeruleus is related to both cognitive decline and severity of motor signs in older adults $(11,12)$. Thus, this has led to the increasing recognition that common neuropathologies and other neurobiologic indices may affect a wider array of clinical domains and manifest more complex clinical phenotypes in older adults (13-15).

The Consensus Group convened by the International Consensus Group (IANA, IAGG) and the International Association of Gerontology and Geriatrics (IAGG), nicely summarized by Kelaiditi et al, represents a timely effort to delineate a complex clinical phenotype which simultaneously captures a broad array of physical and cognitive phenotypes which they termed "cognitive frailty". The panel defined cognitive frailty as a syndrome in older adults with evidence of both physical frailty and cognitive impairment without a clinical diagnosis of AD or another dementia. This new construct extends the prior physical frailty construct described by Fried et al in two ways.16 First, it adds a formal assessment of cognitive function. Second, while the original physical frailty construct only assessed subjective fatigue, the proposed cognitive frailty construct also recommends a more comprehensive assessment of depressive symptoms to capture psychological in additional to physical aspects of fatigue. This may be important because, like the motoric aspects of physical frailty, depressive symptoms are also related to cognitive outcomes $(17,18)$. In addition, depressive symptoms were associated AD pathology in some studies (19) but not others

Corresponding author: David A. Bennett, MD; Rush Alzheimer's Disease Center; Rush University Medical Center; Armour Academic Facility, Suite \#1022; 600 South Paulina Street, Chicago, Illinois 60612; Phone: (312) 942-2362, Fax (312) 563-4604,

David_A_Bennett@rush.edu. 
(20). Further, depressive symptoms are also related to motor function other than fatigue (21). Since motor impairments also can be caused by non-neurologic conditions, the panel hypothesized that the concurrent cognitive impairment in the cognitive frailty construct might provide a means of identifying individuals with cognitive impairment caused by nonneurodegenerative conditions which might be reversible.

Considering both physical frailty and cognition together as a single complex phenotype may have important clinical and research implications. For example, since both physical frailty and cognition predict adverse health outcomes, their cooccurrence is likely to identify people at particularly high risk (22). Geriatric interventions are increasingly multi-modal, targeting cognitive, motor and psychosocial components of aging (23-25). However, it is also likely that some individuals manifesting the proposed criteria for cognitive frailty may be particularly vulnerable due to combined risks and may be less responsive to interventions. Identifying these two groups will require further research.

While the assessment of a complex clinical phenotype has the potential to allow investigators to identify subgroups of individuals with distinctive clinical profiles, these complex phenotypes also have some limitations. To date there are important gaps in our understanding of the inter-relationship between physical frailty and cognition over time. Cognition does not rely directly on structural elements outside the brain. So, non-neurologic causes of cognitive impairment such as those associated with systemic disease must still be mediated through the brain. By contrast, the motor features contributing to physical frailty derive from motor control systems which reside both within the brain as well as in various other CNS regions including the brainstem and spinal cord. Moreover, via peripheral nerves, these motor control systems are integrated together with peripheral musculoskeletal structures and nonneurologic systems such as cardiopulmonary function and systemic metabolism. Thus, it is likely that physical frailty and cognition may show some degree of inter-relationship due to the effect on both from processes occurring in the brain.

Nonetheless the additional differences in their underlying neural pathways may lead to distinctive discrepancies in their trajectories of change over time. Supporting this idea, a recent, study, suggested that post-mortem indices of brain pathologies explain a larger percentage of the variance in declining cognition as compared to the percentage of variance in progressive frailty in the same individuals (26).

It is clear that more longitudinal studies that incorporate cognition, motor function including physical frailty, and psychological constructs such as depression are needed. Extant longitudinal studies which have examined cognitive decline with linear models suggest that on average older adults show cognitive decline. However, the trajectory of change in cognition over long periods of time is not linear. For example, studies using change point models to capture non-linear decline, suggest that several years prior to death there is acceleration in cognitive decline, termed "terminal decline" $(9,10,27)$. Moreover, not only does the rate of cognitive decline differ between the preterminal and terminal segments, but their underlying biology seems to be different. For example, common neuropathologies associated with dementia and physical frailty are more strongly associated with the slope of preterminal cognitive decline than with the slope of terminal cognitive decline. Change in motor function can also be characterized by change point models and terminal decline (28). 
These data underscore the complexity of characterizing the trajectory of a single clinical domain in older adults, let alone a construct with multiple moderately overlapping domains. Not only can the contribution of pathology vary over time i.e., as pathology accumulates, some biologic processes like terminal decline may only contribute during specific stages of aging. So investigators must not only identify risk factors but also the particular stage during which the risk factor may contribute to change in cognition. Thus, decomposing complex heterogeneous phenotypes into smaller components such as physical frailty or cognition can offer insights into common features and facets which might not be apparent if multiple clinical domains are summarized into a single complex clinical phenotype. However, eventually the decomposed clinical components must be reassembled as a range of phenotypes emerge and manifest in aging humans - and since it is at the level of the individual person at which we must ultimately intervene. Thus, we applaud the Consensus Group for their efforts and recognize their important contribution towards synthesizing the extant literature and initiating an approach to cognitive frailty that may bear fruit in the future.

Aging research is complex and needs both efforts, i.e., efforts to develop more complex clinical phenotypes such as cognitive frailty while simultaneously pursuing studies which carefully deconstruct these phenotypes into dissociable components. These complementary research efforts are essential to facilitate the discovery and implementation of effective approaches to improve the health and well-being of the aging population world-wide.

\section{Acknowledgments}

Study funding: Supported by NIH grants P30AG10161, R01AG17917, R01NS078009, R01AG040039, the Illinois Department of Public Health, and the Robert C. Borwell Endowment Fund.

\section{References}

1. Buchman AS, Wilson RS, Bienias JL, Shah RC, Evans DA, Bennett DA. Change in body mass index and risk of incident Alzheimer disease. Neurology. Sep 27; 2005 65(6):892-897. [PubMed: 16186530]

2. Boyle PA, Buchman AS, Wilson RS, Leurgans SE, Bennett DA. Association of muscle strength with the risk of Alzheimer disease and the rate of cognitive decline in community-dwelling older persons. Arch Neurol. Nov; 2009 66(11):1339-1344. [PubMed: 19901164]

3. Buracchio T, Dodge HH, Howieson D, Wasserman D, Kaye J. The Trajectory of Gait Speed Preceding Mild Cognitive Impairment. Arch Neurol. Aug 1; 2010 67(8):980-986. 2010. [PubMed: 20697049]

4. Verghese J, Wang C, Lipton RB, Holtzer R, Xue X. Quantitative gait dysfunction and risk of cognitive decline and dementia. Journal of Neurology, Neurosurgery, and Psychiatry. 2007; 78(9): 929-935. 9/1.

5. Camicioli R, Howieson D, Oken B, Sexton G, Kaye J. Motor slowing precedes cognitive impairment in the oldest old. Neurology. May; 1998 50(5):1496-1498. [PubMed: 9596020]

6. Gustafson D. Adiposity indices and dementia. Lancet Neurol. Aug; 2006 5(8):713-720. [PubMed: 16857578]

7. Wilson RS, Barnes LL, Mendes de Leon CF, et al. Depressive symptoms, cognitive decline, and risk of AD in older persons. Neurology. Aug 13; 2002 59(3):364-370. [PubMed: 12177369]

8. Abellan van Kan G, Rolland Y, Gillette-Guyonnet S, et al. Gait Speed, Body Composition, and Dementia. The EPIDOS-Toulouse Cohort. The Journals of Gerontology Series A: Biological Sciences and Medical Sciences. Apr 1; 2012 67A(4):425-432. 2012. 
9. Buchman AS, Yu L, Wilson RS, Schneider JA, Bennett DA. Association of brain pathology with the progression of frailty in older adults. Neurology. May 28; 2013 80(22):2055-2061. [PubMed: 23635961]

10. Wilson RS, Leurgans SE, Boyle PA, Schneider JA, Bennett DA. Neurodegenerative basis of agerelated cognitive decline. Neurology. 2010; 75(12):1070-1078. [PubMed: 20844243]

11. Buchman AS, Nag S, Shulman JM, et al. Locus coeruleus neuron density and parkinsonism in older adults without Parkinson's disease. Mov Disord. Nov; 2012 27(13):1625-1631. [PubMed: 23038629]

12. Wilson RS, Nag S, Boyle PA, et al. Neural reserve, neuronal density in the locus ceruleus, and cognitive decline. Neurology. Mar 26; 2013 80(13):1202-1208. [PubMed: 23486878]

13. Jack CR Jr. Albert, Knopman DS, et al. Introduction to revised criteria for the diagnosis of Alzheimer's disease: National Institute on Aging and the Alzheimer's Association workgroup. Alzheimers Dement. Apr 20.2011

14. Lang AE. A critical appraisal of the premotor symptoms of Parkinson's disease: Potential usefulness in early diagnosis and design of neuroprotective trials. Movement Disorders. 2011; 26(5):775-783. [PubMed: 21484865]

15. Tanne D, Levine SR. Capturing the Scope of Stroke: Silent, Whispering, and Overt. Archives of Neurology. 2009; 66(7):819-820. [PubMed: 19597082]

16. Fried LP, Tangen CM, Walston J, et al. Frailty in older adults: evidence for a phenotype. J Gerontol A Biol Sci Med Sci. Mar; 2001 56(3):M146-156. [PubMed: 11253156]

17. Saczynski JS, Beiser A, Seshadri S, Auerbach S, Wolf PA, Au R. Depressive symptoms and risk of dementia. Neurology. Jul 6; 2010 75(1):35-41. 2010. [PubMed: 20603483]

18. Barnes DE, Alexopoulos GS, Lopez OL, Williamson JD, Yaffe K. Depressive symptoms, vascular disease, and mild cognitive impairment: findings from the Cardiovascular Health Study. Arch Gen Psychiatry. Mar; 2006 63(3):273-279. [PubMed: 16520432]

19. Rapp MA, Schnaider-Beeri M, Purohit DP, Perl DP, Haroutunian V, Sano M. Increased neurofibrillary tangles in patients with Alzheimer disease with comorbid depression. Am J Geriatr Psychiatry. Feb; 2008 16(2):168-174. [PubMed: 18239198]

20. Tsopelas C, Stewart R, Savva GM, et al. Neuropathological correlates of late-life depression in older people. Br J Psychiatry. Feb; 2011 198(2):109-114. [PubMed: 21282780]

21. Buchman A, Boyle P, Wilson R, et al. Loneliness and the rate of motor decline in old age: the rush memory and aging project, a community-based cohort study. BMC Geriatrics. 2010; 10(1):77. [PubMed: 20969786]

22. Avila-Funes JA, Amieva H, Barberger-Gateau P, et al. Cognitive impairment improves the predictive validity of the phenotype of frailty for adverse health outcomes: the three-city study. J Am Geriatr Soc. Mar; 2009 57(3):453-461. [PubMed: 19245415]

23. Rebok GW, Carlson MC, Langbaum JB. Training and maintaining memory abilities in healthy older adults: traditional and novel approaches. J Gerontol B Psychol Sci Soc Sci. Jun.2007:62. Spec No 1:53-61. [PubMed: 17565166]

24. Cesari M, Vellas B, Gambassi G. The stress of aging. Experimental Gerontology. 2013; 48(4):451456. 4//. [PubMed: 23103391]

25. Subra J, Gillette-Guyonnet S, Cesari M, Oustric S, Vellas B. The integration of frailty into clinical practice: preliminary results from the Gerontopole. J Nutr Health Aging. Aug; 2012 16(8):714720. [PubMed: 23076514]

26. Yu, L.; Buchman, AS.; Boyle, PA., et al. Simultaneous changes in cognition and physical frailty and association of neuropathology.. Paper presented at: Alzheimer's Association International Conference; Boston, MA, USA. 2013;

27. Boyle PA, Wilson RS, Yu L, et al. Much of late life cognitive decline is not due to common neurodegenerative pathologies. Annals of Neurology. 2013 n/a-n/a.

28. Wilson RS, Segawa E, Buchman AS, Boyle PA, Hizel LP, Bennett DA. Terminal Decline in Motor Function. Psych \& Aging. 2012 